\title{
Analysis on the Application of Blue and White Pattern in Clothing Accessories Design
}

\author{
Wei Huang \\ Jiangxi Institute of Fashion Technology \\ Nanchang, China 330201
}

\begin{abstract}
Since the rapid development of arts and culture, the blue and white decorative patters in blue and white porcelain has been widely and flexibleapplied in clothing accessories. By analyzing the application and performance ofthe blue and white porcelain decorative patters in clothing accessories,to see how does Chinese traditional blue and white artistic elements been inherited and innovated in the future design road. Let Chinese traditional culture marching towards the world.
\end{abstract}

Keywords-blue and white pattern; clothing accessories; manifestation; application

\section{INTRODUCTION}

Chinese traditional culture is world-famous by itsextensive knowledge and profound scholarship, blue and white porcelain as the representative of Chinese culture occupies a very important positionin the world history of art. Its beautiful color and pattern, exquisite craftsmanship and rich culture have shocked the whole world. It has a strong cultural charm of Chinese nations, so it became the label of Chinese culture.

\section{THE DEFINITION AND ARTISTIC CHARACTERISTICS OF BLUE AND WHITE PATTERN}

Blue and white pattern first appeared in porcelain which was the blue and white porcelain, the major production place of blue and white porcelain is Jiangxi Jingdezhen. In the "History of Chinese Ceramics" wrote: "Qinghua' refers to the underglaze porcelain which uses the cobalt materials painting on porcelain, then applies the transparent glaze, single firing at a high temperature, and with blue patters". This blue has the features of strong coloration, brilliant color and color stable. Blue and white porcelain not only with rich shapes but alsowith beautiful decorations, it also has national characteristics,it beard the pure and noble feelings of ancients, entrusted with the Chinese nation's spiritual heritage. Blue and white pattern consist with blue and white colors, blue also known as indigo blue. In "Xunzi •Quanxue"it said: "blue, taken from the indigo, butbluer than it." describes it belongs to the blue color system but between blue and green, close to sky blue but a little darker. Blue symbolizes the strong, simple, solemn, hope, noble andplain beauty. It not only gives a comfortable feeling but also gives a wealth association. Traditional Taoist's"Ying Yang Wu Xing theory"believes that the blue color has an auspicious meaning. "White"color is pure and flawless, blue has high purity and with low brightness, so when white matches blue, it will be set off with each other, and prominent the innocence of blue. Thereby not only break the monotony of white but also improve the bright and clear meaning, so that make people feel calm and peace after watch.The pure whitecoupled with calm blue, added with vivid strokes, so that blue and white pattern has infinite charm.

\section{THE MANIFESTATION OF BLUE AND WHITE PATTERN}

Blue and white pattern did not presented as a separate aesthetic subject, it appears to be closely integrated with shapes of decorative objects, texture of the material, technology and other factors. According to their specific application, decorative objects and decorative parts it appeared with the forms of single pattern, two patters repeat, four patters repeat, suitable patterns and corner patters, and integrate with a variety of aesthetic principles in the flexible modelings. Harmony is very important in the design and application of blue and white pattern, harmony contains the "diversity" and "unification" two opposite factors. Diversity means in addition to the decorative object modeling, material and area, it also includes manifestations of blue and white pattern. The current manifestations of clothing accessories patterns can be divided into: flatmanifestation, semi-three-dimensionalmanifestation, three-dimensional manifestation and so on.Flatmanifestation depending on the material features of decorative objects to usespecific technique for manifestation, such as printing, dyeing, embroidery, paintings and so on, the manifestation of semi-three-dimensional and three-dimensional are carving, molding, casting and the like.

\section{The Specific ApPliCATION OF BLUE AND White PATTERN IN CLOTHING ACCESSORIES DESIGN}

The application of blue and white pattern in clothing accessories design fully considered the modern art style, aesthetics, consumer culture, socio-economic and other factors, and on premise of considering specific application object, combine the traditional patterns together with popular information of the times, traditional designs with a new gesture to display in modern clothing accessories. Express the typical elements of traditional patterns through figurative and abstract forms, but also integrate withall kinds of clothing accessories features, use constitute idea's dot, line, surface and other brand new modeling techniques to make the design in order to make it suitable for different objects. 
A. The Application of Blue and White Patterns in Bag Design, Bagsare One of the Important Clothing Accessories at the Moment, They Are the Essential Goods of Our Life

Of course besides its functionality it also has artistry. The application of blue and white pattern in bags is based on the style, classical style bags usea large number of blue and white patterns which implies rich, noble, longevity, happiness and other auspicious patterns. Chinese traditional decorative patter stress "picture must has meaning, meaning must be auspicious", which is the reason why traditional pattern spread so far and also is the new bright spot injected by traditional patternsto modern design. Such as lotus pattern which convey auspicious ; peony pattern as the symbol of wealth and auspicious; chrysanthemum pattern as the symbol of longevity and noble sentiments, as well as banana leaf pattern, pine, bamboo and plum pattern, Baoxiang pattern and the like. There also are dragon and phoenix patterns as the symbol of imperial power and beauty, as well as fish pattern, lion patter and so on. There are some applications of auxiliary patters such as: Turtle back pattern, cloud patter, Turtle pattern, back-shaped pattern, moonpatter and curly grass pattern. Some styles absorb Western art concepts apply with some abstractblue and white patterns such as: blue and white plaid patter composed by dot, line and surface, blue and white Persian patterns, also use some simple character modelings, landscape, painting, art text blue and white patterns which affected by Pop Art . Overall, the application of blue and white pattern in bags are relatively free, in addition to material limit on performance, others can makearbitrary play. The materials of bags are generally divided into fabric and leather, despite leather appeared withsemi-three-dimensional carved and hollow forms, the othersare the same with fabric material mostly in the form of flatmanifestation, such as: digital printing, screen printing, thermal transfer, dyeing, embroidery and directlydrawn. Decorative parts include center, edge, corner and full floral decoration. Central decorations generally are single patter and appeared as suitable patters; edge decoration normally are two patter repeat form; if it is full floral decoration, then it normally use four patter repeat form; corners decorations normally appeared withcorner patters.

\section{B. Theapplication of Blue and White Pattern in Footwear, Hats and Fans Is Similar with Bags}

Thepatters also divided into traditional and epidemic style.Traditional style generally use animal and plant symbols as themes to express certain meaning, and the design and application of epidemic styleblue and white pattern is freer. The application of blue and white pattern on the footwear is different from the bags, it should design the patter according to the decorative position.Footwear decorations general on the upper and vamp, shape design should according to its specific location, in the upper of the shoe is usually two patterns repeat blue and white patter, on the vamp normally design the suitable blue and white patter according to thevamp change. If the vampis fabric material,thenafter cutting uselocating digital printing, screen printing, heat transfer and embroidery patters for decoration; some may directly drawn the blue and white patters with textile pigment or acrylic paint after the footwearfinished, since the stroke change and occasionality, it makes blue and white patternswith more artistic quality. And the design of blue and white patters in hats and fans sometimes required the patter need to fit the shape of the hat and fan, but generally the applications are relatively free. The scope of its subject matter is relatively wide range and free. The patter production process is similar with bags and footwear.

\section{Necklace, Bracelet and Bangle Are Important Accessories to Decorate Body, They Also Have A Large Number of Blue and White Patterns for Decoration}

Blue and white pattern generally appeared in the form of beads necklace, pendant and card. Beads necklaces, pendants and cards all firing with modeling polymer clay, and then draw through the form of overglaze and underglaze. Necklace is a symbolic expression of personality, different textures, different forms, different colors pendant express women's personality and taste. In order to produce luxury exquisite necklace, it added the precious material pendant based on blue and white porcelainto expresses luxury orientation; added wooden pendant to expresses the simple, natural, flexible orientation; added crystal pendant to express fresh, crisp, crystal clear orientation. When matching the materials, we should consider the decorative effect, for example if there is a pendant or other accessories then blue and white pattern should design on them, we could inlay the fired blue and white into the metal and the pendant could use polymer clay for three-dimensional modeling, in order to exhibit blue and white patterns, the carefully design,the artistic patter and the modern styling.

\section{Earrings and Rings}

Earrings have the function of finishing touch and make up facial defects. There are many styles and production methods for earrings, generally divided into insert type and clamp type; in styles there are circular, square, triangular shapes, and all sorts of irregular geometry, ever-changing. Application of blue and white pattern on the earrings normally use polymer clay as the carrier and use silver, gold, alloy or other metals as the ear hook or ear clamp; then use metal for the ear nail bracket; porcelain pieces with blue and white patterns inlaid or fixed on the bracket. Some simple blue and white earrings can prick holes on all kinds of polymer clay, and directly connect them with small metal rings and ear hooks, blue and white pattern on the polymer clay can make free performance. The ring is a small circle on the finger, usually made of metal and jade. The application of blue and white in rings mainly appeared as inlay, mostly inlays are fired by polymer clay modelings, then inlay the fired blue and white modeling into the metal. Threedimensional modeling can make the blue and white patter through paste method or slice pattern, also we can create threedimensional flower performance.

\section{E. Application of Hairpins and Buttons}

The application of blue and white pattern in hairpin are mainly hairpin pendants, hairpin pendants is small-sized but it has enormous changes, blue and white pattern can be applied to objects with different shapes, andit can also be applied on the sheet-like objects. There are many pendant materials but for blue and white patters they usually use clay and cloth, planar pattern generally produced by printing and dyeing and painting,for now in order to raise the efficiency and reduce the 
costs, they firing after decal on the ceramics pieces. For threedimensional blue and white patterns, first use polymer clay for modeling then draw the blue and white pattern.Button is very important clothing accessories, it has different colors and materials. If the button is bamboo, wood and shell materials, then the blue and white patternappeared with printing and dyeing forms, and ceramic material can be fired with polymer clay into exquisite blue and white pattern. And buttons with different shapes and blue and white patterns have certain function with theformation of overall clothing style: such as children's clothing coulduse cartoon shaped or blue and white pattern buttons in order to make the clothing full of childishness.

\section{F. Mobile Phone and Lighter}

With the development of the times and the progress of technology, mobile phone has become a new clothing accessories.In order to pursuit artistry and cultural connotation; designers make new designs onphone shell and phone chain, so the design and application of blue the white pattern have a new development space. First, polymer clay will not scratch the phone like some other materials.Roped up some Polymer Clay Beads with blue and white patterns will add unlimited charm to the phone. And also it can be roped up with other material beads or gemstones, the effect will be better, not only improves the quality but also forms a contrast with the blue and white pattern. The blue and white patterns on phone shells and lightsare normally on the object body, the design patterns are more flexible and fashion, but we need to consider the size and the shape of the object.

\section{CONCLUSION}

From the perspective of art development, any kind of art in order to have a strong vitality, it must be "open development, fully inclusive and equitable". The current application of blue and white pattern in clothing accessories shows a diverse design style and situation, our heritage and innovation were built on the spirit of traditional culture connotation, so the blue and white pattern should based on the spiritual connotation of tradition heritage and rich meanings to design new patterns to meet people's aesthetic needs. First of all, do not blindly "brought", we need to select, refine and re-combination from the traditional patters in order to produce a new meaning. Secondly, on the pattern expression methods, in addition to traditional ceramic firing, decals, printing and dyeing, we should add newexpression methods such like: 3D printing, weaving, embroidery, and also integrate the texture and thickness variation of the materials into the application.On the application of blue and white pattern, we should stick withblue an white color application, enhance color interspersed changes and its sense of color composition, so that to enhance the expression of the design content and theme. Finally, when use the blue and white pattern we should focus on the application of symbol meanings, traditional Chinese decorative patterns attaches great importance to the "meaning" expression, blue and white pattern as an important element of traditional Chinese culture, it will help enhance the sense of Chinese elements, also let people feel our profound Chinese culture. In this way the application of blue and white pattern in clothing accessoriesgives the cultural connotation and artistic value to the accessories works. Chinese designers should rely on profound traditional cultural heritage to spread and promote Chinese culture and spirit.

\section{REFERENCES}

[1] Zhang Jingjing. Fashion ceramics.Clothing Accessories [M] Wuhan: Wuhan University of Technology Press .2006.05.

[2] Harry Ghana. Oriental blue and white porcelain [M]. Translated by Ye Wencheng,Luo Lihua. Shanghai People's Fine Arts Publishing Company, 1992.

[3] Zheng Jun, Yuan Hong, etc.Decoration Art of Chinese blue and white porcelain [M]. People's Fine Arts Publishing Company, 2010,01.

[4] Huang Yunpeng. Decorative characteristics of Folk blue and white porcelain [M]. Jingdezhen ceramics, 1991.

[5] Hu Meisheng. Style characteristics and decorativelaws of the blue and white art [M].Ceramics, 1980,04. 